\title{
Protocolo de Atendimento Psicológico em um Serviço de Reprodução Humana Assistida do Sistema Único de Saúde - SUS
}

\author{
Mara Farias Chaves Vieira, ${ }^{1, *}$, \& Maria Liz Cunha de Oliveira ${ }^{2}$ \\ 'Secretaria de Estado de Saúde do Distrito Federal, Brasília, DF, Brasil \\ ${ }^{2}$ Escola Superior de Ciências da Saúde, Fundação de Ensino e Pesquisa em Ciências da Saúde (ESCS/FEPECS), \\ Brasília, DF, Brasil
}

\begin{abstract}
RESUMO - Este trabalho apresenta uma solução otimizada para atender casais submetidos a tratamentos de infertilidade em um serviço público. O objetivo foi a construção de um protocolo de atendimento psicológico. A metodologia foi baseada em técnicas da pesquisa operacional, sendo o protocolo elaborado em cinco etapas: 1) construção do fluxograma de atendimento; 2) elaboração do instrumento de entrevista semiestruturada; 3) aplicação de instrumentos de avaliação; 4) criação do grupo de apoio aos casais; 5) estabelecimento da rotina de atuação da psicologia. Como resultado, apresenta-se o protocolo criado. A criação desse protocolo busca contribuir para a necessária sistematização da entrevista psicológica, melhorando a qualidade da assistência integral a esses casais.
\end{abstract}

PALAVRAS-CHAVE: psicologia da saúde, infertilidade, avaliação psicológica, tecnologia reprodutiva, pesquisa operacional

\section{Psychological Assistance Protocol in a Human Reproductive Service of Brazilian Unified Health System.}

\begin{abstract}
This paper presents an improved solution for the care of couples undergoing infertility treatments in a public service. The purpose of the study was to develop a psychological treatment protocol. The methodology used was based on techniques of operations research and protocol elaborated of five steps: 1) construction of service flowchart; 2) preparation of semi-structured interview tool; 3) application of assessment tools; 4) creation of a support group for couples;; 5) establishment of psychological intervention routine. As a result the elaborated protocol is presented. The creation of this protocol aims to contribute to the necessary systematization, improving the quality of comprehensive care to these couples. KEYWORDS: health psychology, infertility, psychological evaluation, reproductive technology, operational research
\end{abstract}

A constituição de uma família e o tornar-se pai e mãe são fenômenos importantes para o estabelecimento de uma identidade social adulta, o que contribui para a realização pessoal do indivíduo (Dornelles, 2009). Entretanto, é comum na atualidade as pessoas adiarem o projeto parental em busca de uma realização profissional, de um melhor status socioeconômico ou mesmo aguardando o encontro do cônjuge ideal. Quando finalmente decidem ter filhos, não é raro que os casais se deparem com um declínio na capacidade reprodutiva da mulher ou do homem, capacidade em potencial somente confirmada com a gestação. Assim, a infertilidade pode ser uma realidade para esses casais, um diagnóstico frequentemente associado a impotência, culpa, vergonha e fracasso (Dornelles, 2009).

AAmerican Society for Reproductive Medicine (ASRM, 2013) define como infertilidade a ausência de gestação após 12 meses de relações sexuais frequentes (duas a três por semana) sem anticoncepção. A Organização Mundial de Saúde reconhece a infertilidade como um problema de Saúde Pública que afeta de 8 a $12 \%$ dos casais em todo o mundo. No entanto, a infertilidade não deve ser entendida como uma doença clássica, pois nem sempre é acompanhada de componentes como dor, internação e risco de vida, mas, ainda assim, ela desencadeia diversas alterações psicológicas, sendo entendida como uma situação

*E-mail: maracvieira@gmail.com 
ameaçadora e geradora de diferentes conflitos, sentimentos e sensações (Melamed, 2013).

Klonoff-Cohen et al. (2001) sustentam que a infertilidade é vivida como um evento traumático para a maioria dos casais, sendo experienciada como o acontecimento mais estressante de suas vidas. A incapacidade de conceber pelos métodos naturais gera uma ferida narcísica, suscitando sentimentos dolorosos e vivências intensas (Dornelles, 2009), podendo causar um estado de depressão ou outros transtornos emocionais, muito embora devam ser vistas pelos profissionais como reações normais frente a uma situação anormal, que é a infertilidade (Seger \& Melamed, 2011). O sujeito se vê impossibilitado de dar continuidade à própria existência através de gerações futuras, de ressignificar sua própria história de filho, sob a perspectiva de progenitor e de preencher possíveis vazios pessoais e familiares através da parentalidade (Balmaceda et al., 2001).

Assim, compreendendo a infertilidade como uma situação potencialmente traumática de vida, que inclui questões socioculturais, psicológicas e espirituais importantes, o papel do psicólogo, através de acompanhamento psicoterápico breve/focal, entre outros, deve ser o de: 1) contribuir com sua escuta e acolhimento do paciente, ajudando-o a lidar de forma menos dolorosa com os desdobramentos da infertilidade e tratamento; 2) facilitar o enfrentamento dos medos, angústias e mitos, ofertando informação e possibilitando o reconhecimento de suas reais possibilidades frente aos recursos oferecidos pela medicina; 3 ) minimizar o estresse provocado pela infertilidade e tratamento através da potencialização dos recursos internos (capacidades adaptativas) do casal para lidar com o estresse, dotando esses pacientes da melhor qualidade de vida possível (Melamed, 2013).

Diante dessa realidade, as técnicas de reprodução assistida (TRA) surgem como alternativas de solução para o problema da infertilidade. As buscas por esses tratamentos são compreendidas como movimentos adaptativos dos sujeitos, no sentido de superar o problema. Seger (2009) destaca que existem diferenças de timing dos casais: alguns buscam tratamento imediato assim que surgem os primeiros problemas, outros são mais ponderados e um terceiro grupo não busca em nenhum momento um tratamento específico. Há ainda aqueles que iniciam, param por um tempo e voltam a procurar tratamento após meses ou anos. Assim, a forma do casal vivenciar e lidar com o problema da infertilidade ocorre de inúmeras maneiras e cada uma delas mostra indícios dos traços de personalidade de cada um, como chegam ao serviço e o que esperam da equipe.

Desse modo, as clínicas/serviços de Reprodução Humana Assistida (RHA), oferecem esperança a esses casais em busca da concretização da parentalidade. O Centro de Estudos e Pesquisa em Reprodução Assistida (CEPRA) nasceu em 1998, na Secretaria de Estado de Saúde do DF, a partir da compreensão de que o planejamento familiar não corresponde apenas ao controle de natalidade, mas também ao direito que o cidadão tem de programar o momento de ter filhos.

O centro oferece os serviços de Inseminação Intra Uterina (IIU) e Fertilização In Vitro (FIV) pelo Sistema Único de Saúde (SUS) para casais inférteis e localiza-se dentro do Setor de Reprodução Humana (SRH) do Hospital Materno Infantil de Brasília - HMIB. O CEPRA oferece um serviço mais abrangente, que engloba o acompanhamento das pacientes no climatério, os serviços de endoscopia e endocrinologia ginecológica e o tratamento da infertilidade.

Para tratamentos de RHA, são atendidos, em média, 30 a 35 casais por mês e o perfil da população atendida são indivíduos com infertilidade primária (nunca tiveram filhos) ou secundária (já tiveram filhos e depois apresentaram problemas de fertilidade).

Pelo próprio princípio da universalidade do SUS, para usufruir desse serviço não há limite de idade, condição financeira ou nível educacional. A maioria dos pacientes reside no DF ou entorno, embora usuários de outros estados também procurem o serviço eventualmente.

A equipe do centro é composta por dez médicos ginecologistas, uma médica geneticista e um urologista, três biólogos, uma psicóloga, uma enfermeira, três auxiliares de enfermagem, uma técnica administrativa e a coordenadora do serviço. O casal que necessita do tratamento em RHA precisa vir referenciado por um serviço de atenção primária ou secundária (geralmente centros de saúde ou hospitais gerais), já tendo realizado exames e com um diagnóstico de infertilidade.

Ao chegar ao SRH, o casal passa por uma primeira consulta no ambulatório de infertilidade, onde levará todos os exames já realizados. Após análise dos exames e avaliação clínica de qual tratamento é o mais adequado de acordo com a(s) causa(s) da infertilidade, o casal será inscrito na fila de espera para IIU ou FIV, quando recebem um número, através do qual poderão acompanhar o andamento da fila. Quando chegar a vez do casal, esse é convocado pela equipe do CEPRA através de contato telefônico. O tempo médio de espera para IIU é de até 1 ano e para FIV é de 3 anos e meio.

É nesse contexto que surge a necessidade não só da atuação, mas também da padronização do trabalho do psicólogo. Uma das formas de objetivar essa prática é através do uso de protocolos, por meio dos quais elencamse algumas prioridades, distribuídas em procedimentos, recomendações e encaminhamentos (Prosdocimo, 2011).

Assim, o presente estudo tem por objetivo construir um protocolo de atendimento psicológico aos casais atendidos no CEPRA. A criação desse protocolo busca contribuir para essa necessária sistematização, melhorando a qualidade da assistência integral a esses casais. 


\section{MÉTODO}

A psicóloga da Secretaria de Estado de Saúde (SES-DF) já atuava no serviço de reprodução humana (SRH) do Centro de Estudos e Pesquisa em Reprodução Assistida (CEPRA), elaborando pareceres psicológicos solicitados pelos médicos antes do início do tratamento de cada casal. No entanto, a mesma não era integrada à equipe e não conhecia bem o funcionamento do serviço, atuando de forma isolada.

Ao cursar a disciplina "Práticas em serviço", do Mestrado Profissional em Ciências da Saúde da Fundação de Ensino e Pesquisa em Ciências da Saúde (FEPECS), a psicóloga passou $60 \mathrm{hs}$ atuando no serviço, no qual desenvolveu a pesquisa, objetivando levantar as demandas dos profissionais e compreender melhor o perfil e necessidades dos usuários, de modo a refletir sobre a prática e propor soluções. Graças a esse levantamento, foi possível a elaboração do protocolo. Dois profissionais do CEPRA (a coordenadora do serviço e um dos biólogos) se propuseram a ser supervisores/orientadores da psicóloga, tendo sido bastante receptivos e facilitando o acesso aos prontuários dos usuários e aos arquivos do CEPRA.
O protocolo de atendimento da psicologia foi estruturado tendo como diretrizes: a busca pelo atendimento integral à saúde do usuário, promoção do diálogo contínuo com a equipe do CEPRA e a construção da interdisciplinaridade, tendo o casal em tratamento como foco.

A construção do protocolo se fundamentou nos objetivos da Psicologia da Saúde (Divisão 38) da American Psychological Association (APA), que tem como foco: (1) utilizar contribuições avançadas da psicologia para compreender o processo saúde-doença por meio de pesquisa básica e clínica; (2) encorajar a integração da informação biomédica com conhecimento psicológico atual; (3) promover educação e serviços em Psicologia da Saúde e (4) informar a comunidade psicológica, biomédica e o público em geral sobre os resultados de pesquisas recentes e atividades de serviço nessa área.

O protocolo desenvolvido foi baseado em técnicas da pesquisa operacional, considerando o fluxo de pacientes e atendimento psicológico.

\section{RESULTADOS}

Foi criado um protocolo que se estruturou em cinco etapas: (1) construção do fluxograma de atendimento do CEPRA; (2) elaboração do instrumento de entrevista semiestruturada; (3) aplicação de instrumentos de avaliação; (4) criação e encaminhamento de casais ao Grupo de Apoio ao Paciente em Reprodução Assistida (GAPRA) e (5) estabelecimento da rotina de atuação da psicologia e melhoria contínua do protocolo a partir da revisão periódica do mesmo.

\section{$I^{\text {a }}$ etapa - Construção do Fluxograma de Atendimento do CEPRA}

A construção do fluxograma de atendimento do CEPRA (Apêndice A) foi possível a partir do conhecimento da rotina e levantamento das necessidades do serviço, o que se efetivou por meio da articulação institucional, contato com a equipe e escuta dos usuários do serviço.

A articulação institucional consistiu no apoio da coordenadora do CEPRA por meio da apresentação da psicóloga à equipe, convite e participação dessa nas reuniões semanais de equipe e facilitação do acesso aos prontuários dos usuários e aos arquivos do CEPRA.

O contato com a equipe se efetivou pelo acompanhamento e observação das rotinas do serviço, entrevistas com os profissionais atuantes, participação nas reuniões de discussões de caso e apresentação de artigos científicos e escuta das expectativas desses profissionais em relação à atuação da psicologia. Além disso, procedeu-se à escuta dos usuários do serviço, através de entrevistas e da observação da socialização dos mesmos na sala de espera.

Todos esses passos facilitaram uma compreensão efetiva do funcionamento do serviço e das etapas dos tratamentos realizados, possibilitando a criação do fluxograma.

\section{$2^{a}$ etapa - Elaboração do Instrumento de Entrevista Semiestruturada}

Como não existia um protocolo de entrevista nesse serviço, a segunda etapa consistiu na elaboração do instrumento de entrevista semiestruturada para o casal (Apêndice B). Tavares (2000) sustenta que as entrevistas semiestruturadas são assim denominadas porque o entrevistador tem clareza dos seus objetivos, de quais informações são necessárias para atingi-los, de como essas informações devem ser obtidas (perguntas padronizadas) e como devem ser consideradas (utilização de critérios de avaliação). Elas costumam ser de grande utilidade em settings ${ }^{1}$ onde é importante a padronização de procedimentos e registros de dados, como na saúde pública e na psicologia hospitalar, que é o nosso contexto.

A elaboração desse instrumento se deu a partir de um levantamento das necessidades da equipe e dos usuários, tendo como diretrizes: estar coerente com a instituição como um todo, integrar-se às rotinas da equipe e centrar o trabalho da psicóloga nas necessidades dos casais, atuando

1 Setting: No contexto da psicologia, refere-se ao local físico onde ocorre o processo terapêutico 
resolutivamente nas dificuldades específicas de cada casal atendido. A entrevista deve ocorrer num primeiro contato da psicóloga com o casal e seu objetivo deve ser, em última instância, compreender como o casal está emocionalmente no momento da entrevista e como ele lida com a infertilidade.

Em sua estrutura, essa abordagem inicial visa colher as seguintes informações do casal: 1) dados sociodemográficos; 2) anamnese da história reprodutiva do casal; 3) estado emocional no momento da entrevista; 4) contexto sociofamiliar e 5) exame do estado mental.

Na coleta dos dados sociodemográficos básicos do casal, as variáveis coletadas são: nome, idade, data de nascimento, naturalidade, atividade profissional, escolaridade, religião e local de residência. Esses dados fornecem ao psicólogo o perfil do casal que procura os serviços do CEPRA, seu nível socioeconômico e também podem ser úteis para futura pesquisa em Psicologia da Saúde.

A anamnese da história reprodutiva do casal consiste na investigação de aspectos como infertilidade passada e atual, passado obstétrico/ginecológico da mulher, tratamento a ser realizado e tempo na fila de espera, filhos (se existem, se são do casal ou de apenas um dos parceiros, adotados, de outros relacionamentos ou de tratamentos anteriores), tratamentos realizados anteriormente e seus desdobramentos.

Quanto à avaliação do estado emocional do casal, observamos: grau de ansiedade, quais os fatores geradores de estresse e que recursos utilizam para controle/manejo do mesmo, predisposição aos estados depressivos, se possuem antecedentes psiquiátricos e se fazem uso de psicotrópicos e outros fármacos utilizados. Observa-se se há queixas, sejam elas ligadas ou não ao tratamento, como por exemplo: demora em realizar o sonho da maternidade/paternidade, angústia diante da incerteza do prognóstico, dificuldades de adesão ao tratamento, pressão social para ter filhos, elevado contingente de exames, elevado tempo de espera na fila da FIV, entre outras.

Ao avaliarmos as condições familiares e sociais do casal observamos o contexto sociofamiliar em que a infertilidade está presente e como ela interferiu na relação do casal, a estrutura familiar, as possíveis crenças oriundas das famílias de origem de cada parceiro, se possuem uma rede de apoio social satisfatória, se resolveram contar ou não sobre a infertilidade e tratamento em seus diversos contextos sociais, etc.

Por fim, realizamos um breve exame do estado mental, ou seja, uma avaliação das funções psíquicas básicas do casal, como consciência, atenção, orientação, pensamento, linguagem e afetividade. Observamos se o mesmo responde às perguntas por meio do relato verbal, considerando também a expressão não-verbal, de acordo com o roteiro específico de perguntas e recomendações desenvolvidas por Dalgalarrondo (2008). Esse autor pontua que a avaliação das funções psíquicas deve ser conduzida com habilidade pelo psicólogo priorizando a escuta e observação do paciente, sendo importante procurar manter contato visual à medida em que estabelecemos um vínculo com o casal.

Nos casos dos pacientes colaborativos, que se expressam bastante durante a entrevista, esse exame do estado mental é feito à medida que eles respondem às questões da entrevista semiestruturada, pois as várias funções avaliadas são áreas de informação tão integradas que se torna mais produtivo não separá-las na prática, completando os dados da entrevista com perguntas suplementares (Cunha, 2000). Nos casos dos pacientes pouco colaborativos, que não desenvolvem um discurso contínuo, apenas respondendo às perguntas que fazemos, escolhemos aplicar o Mini-Exame do Estado Mental (MEEM) mais conhecido como "Mini-Mental" (Folstein, Folstein, \& McHugh, 1975).

Quando esse exame indicar importantes alterações nas funções psíquicas, deve-se comunicar a equipe de saúde e encaminhar para uma avaliação psiquiátrica. É importante salientar, no entanto, que homens e mulheres em tratamento para infertilidade, em geral, são pessoas que têm uma saúde mental que não difere da população geral (Santos, Lanius, \& Makuch, 2013).

Outras questões investigadas na entrevista são: os projetos de vida do casal e o nível de compreensão do tratamento, suas implicações e necessidades observadas. A avaliação sobre o nível de compreensão do tratamento e das taxas de sucesso é particularmente útil para se ter uma ideia das expectativas do casal, de forma que se possa corrigir percepções equivocadas acerca das reais possibilidades do tratamento. Também é útil para saber se o casal está consciente das etapas posteriores do tratamento, o que ajuda a gerar uma boa adesão e participação nos procedimentos necessários.

Essa primeira entrevista é, principalmente, avaliativa. Entretanto, algumas intervenções podem ser feitas de acordo com as necessidades do casal. Por exemplo, quando surgem dúvidas durante a entrevista, a psicóloga procura esclarecê-las e, no caso de dúvidas mais específicas ou ligadas especificamente ao diagnóstico do casal, o mesmo é incentivado a buscar a resposta com outros profissionais da equipe. Os tipos de dúvidas apresentadas também nos revelam o grau de conhecimento do casal acerca de seu diagnóstico e tratamento. É importante lembrar que os pacientes que se submetem aos procedimentos de RHA necessitam de informações que contribuam para reduzir o estresse e a ansiedade gerados pela infertilidade e tratamentos (Travain, Lourenço, \& Tardin, 2013).

Também é realizado, quando necessário, aconselhamento psicológico dos casais, compreendido aqui como uma intervenção psicológica de apoio. Trabalham-se as possibilidades de mulheres e homens de lidarem efetivamente com a situação da infertilidade, procurando mobilizar seus recursos internos de enfrentamento. Da mesma forma, quando surgem conflitos no casal, o psicólogo media tais conflitos procurando favorecer a expressão dos sentimentos e facilitar a tomada de decisões. Caso não seja possível 
realizar todas as intervenções necessárias nesse primeiro atendimento, outros serão agendados para acompanhamento psicológico do casal, até que as principais demandas psicológicas referentes à infertilidade e/ou tratamento dessa sejam trabalhadas. Caso surjam demandas que ultrapassem o referido contexto, o casal será encaminhado para acompanhamento psicológico externo.

$\mathrm{O}$ conjunto de dados obtidos nessa primeira fase nos fornece uma visão panorâmica acerca do casal em tratamento e gera um parecer psicológico, fundamentado numa breve descrição dos aspectos relevantes observados durante a entrevista. Esse parecer responde se o casal está "apto" ou "não apto" para a realização do tratamento. São utilizados os seguintes critérios, de exclusão ou postergação do tratamento, indicados pela ASMR: psicopatologia severa (quando detectadas importantes alterações nas funções psíquicas), transtornos psicológicos preexistentes que o tratamento ou gravidez possam agravar, discórdias conjugais graves, coerção de um membro do casal, abuso de substâncias e determinadas situações críticas, particulares a cada indivíduo.

O parecer psicológico nos fornece uma compreensão sobre quais recursos o casal dispõe para lidar com suas dificuldades, sua resiliência e seus recursos adaptativos, se há necessidade ou não de continuidade dos atendimentos psicológicos e quais encaminhamentos devem ser feitos.

É importante salientar que, na comunicação dos resultados dessa avaliação psicológica à equipe multidisciplinar, o psicólogo deve guiar-se sempre pelos preceitos de seu código de ética profissional e divulgar apenas as informações necessárias ao andamento do tratamento, buscando proteger a privacidade do casal atendido (Travain, Lourenço, \& Tardin, 2013).

\section{$3^{\text {a }}$ etapa - Aplicação de Instrumentos de Avaliação.}

Aos pacientes pouco colaborativos, escolhemos complementar a avaliação das funções psíquicas com a aplicação do Mini-Exame do Estado Mental (MEEM). Trata-se de um instrumento de rastreio de comprometimento cognitivo bastante utilizado mundialmente, possuindo versões em diversas línguas e países e já validado para a população brasileira. Seu escore pode variar de 0 a 30 pontos, onde 0 indica o maior grau de comprometimento cognitivo dos indivíduos e 30 a melhor capacidade cognitiva.

O MEEM foi projetado para ser uma avaliação clínica prática de mudança do estado cognitivo em pacientes geriátricos (Chaves, n.d.). Esse exame avalia a orientação temporal e espacial, memória de curto prazo, habilidades de linguagem, entre outras funções cognitivas. Aqui é usado como teste de rastreio e avaliação cognitiva, visando detectar possíveis déficits. Sendo detectado um déficit cognitivo significativo, do ponto de vista da psicologia, desaconselhamos a continuidade do tratamento, uma vez que esse é repleto de orientações sobre medicações, inclusive injetáveis, com horários e dosagens bem específicas, em que um mínimo de compreensão é exigido.

A situação de infertilidade pode gerar um elevado nível de ansiedade e até mesmo quadro depressivo, em função da frustração, do desgaste e das perdas. No entanto, quando é detectado graus elevados de ansiedade e/ou humor deprimido, aplicamos o Inventário de Ansiedade Beck (BAI) e o Inventário de Depressão Beck (BDI; Cunha, 2011). O objetivo ao aplicar essas escala é avaliar o grau desses sintomas (mínimo, leve, moderado ou grave), de forma a corroborar e mensurar o que foi detectado na entrevista.

Em caso de escore elevado, caracterizando ansiedade e/ ou depressão "graves" (de 31 a 63 pontos para BAI e de 36 a 63 pontos para $\mathrm{BDI}$ ), o paciente será encaminhado para atendimento psiquiátrico e será desaconselhada, pelo menos temporariamente, a realização do tratamento.

\section{$4^{a}$ etapa - Criação do Grupo de Apoio ao Paciente em Reprodução Assistida (GAPRA) e Encaminhamento dos Casais.}

O grupo de apoio tem por objetivos: 1) fornecer informações de forma a reduzir o estresse e sofrimento vivenciados pelos casais; 2 ) oferecer apoio através da troca de experiências.

O grupo será composto pelos casais inscritos no CEPRA, tanto pelos casais que aguardam ser chamados nas filas para IIU ou FIV, quanto por aqueles que já se estão sendo atendidos, que poderão participar se desejarem. Entretanto, os casais que tiverem indicação da psicóloga após a entrevista/ avaliação inicial deverão participar como condição para continuidade do tratamento. Apenas o primeiro encontro do grupo será obrigatório para todos os casais já convocados, pois é nesse primeiro encontro que o tratamento é explicado pela equipe multidisciplinar (psicóloga e biólogos) e as dúvidas são dirimidas. Dessa forma, o primeiro encontro terá um caráter primordialmente educativo.

Os demais encontros configuram o grupo de apoio propriamente dito. Aqui a palavra é aberta aos casais e estimula-se o compartilhar das experiências e sentimentos vivenciados nessa busca pela realização da maternidade/ paternidade. O grupo será homogêneo (todos os participantes se encontram na mesma situação de busca de tratamento para infertilidade) e conduzido pela psicóloga, terapeuta do grupo. Os encontros promoverão, assim, a oportunidade de intercâmbio de experiências e informações por pessoas que estão vivendo experiências semelhantes, ajudando o casal a lidar melhor com a infertilidade e o tratamento e empoderando-os para tornarem-se cada vez mais agentes de suas escolhas. 


\section{$5^{\text {a }}$ etapa - Estabelecimento da Rotina de Atuação da Psicologia e Revisão Periódica do Protocolo a Partir da Prática no Serviço.}

O protocolo de atendimento psicológico aos casais do CEPRA seguirá o fluxograma constante no Apêndice C. A revisão periódica do protocolo será feita a partir das necessidades práticas do serviço e dos usuários. Isso confere um caráter não-estático ao protocolo, permitindo e incentivando o revisar constante dos processos de trabalho, conforme novas demandas forem surgindo.
A proposta é que o protocolo seja revisto a cada 6 (seis) meses pela psicóloga em conjunto com a equipe do CEPRA, sempre tendo como foco os usuários do serviço, adaptando-se às possíveis novas demandas e possibilitando o surgimento de novas versões do protocolo.

Todos os participantes e colaboradores desse estudo assinaram o Termo de Consentimento Livre e Esclarecido, estando cientes dos objetivos do trabalho e da manutenção do sigilo.

\section{CONSIDERAÇÕES FINAIS}

A criação de um protocolo proporciona a sistematização necessária à atuação do Psicólogo da Saúde no contexto de um serviço de reprodução assistida, de modo a coordenar tal atuação com ações e intervenções planejadas, integradas e coerentes com a instituição onde se insere e gerar resultados avaliáveis. Além disso, a avaliação psicológica realizada nesse serviço nos ajuda a identificar a percepção dos casais sobre infertilidade e as repercussões psicológicas provocadas por ela.

Nesse serviço, a criação desse protocolo seguiu um processo de construção criativa e inovadora para o estabelecimento de ações, intervenções e procedimentos antes não estruturados nem definidos. Essas ações foram fundamentadas em dados e evidências levantadas, exigiram autocrítica, experimentação, reavaliações permanentes e geraram um protocolo, embasado cientificamente.

A elaboração do instrumento de entrevista semiestruturada para atendimento/avaliação inicial, direciona a coleta de informações do psicólogo necessárias ao atendimento integral ao casal em tratamento de RHA e guia as ações do mesmo com base nessa mesma coleta de dados, de acordo com as necessidades desses pacientes. Isso possibilitou ao Psicólogo da Saúde a emissão de um parecer psicológico, que descreve o estado psicoemocional atual do casal. Com o uso desse protocolo, pode-se avaliar possíveis fatores que prejudicarão o processo de RA.

A curto prazo, o protocolo proporciona a própria organização do serviço, potencializando a efetividade dos atendimentos e dando visibilidade ao papel do psicólogo no contexto das equipes atuantes e da instituição. A médio prazo, espera-se que a sistematização da assistência e o banco de dados gerados pelo protocolo facilite a pesquisa. Não nos referimos exclusivamente aos projetos de pesquisa interinstitucionais, mas também aos pequenos levantamentos do próprio serviço, construindo uma cultura interna de autoavaliação e revisão periódica dos processos de trabalho, proporcionando um caráter dinâmico e autoavaliativo ao protocolo. A longo prazo, o papel do Psicólogo da Saúde no CEPRA contribuirá para uma maior compreensão do processo saúde e doença e promoverá educação, comunicação e eficiência dos serviços de saúde como um todo.

Do ponto de vista dos usuários do serviço, o atendimento psicológico centrado em suas necessidades facilita uma ressignificação da vivência da infertilidade, ajudando-os a lidar melhor com suas repercussões e potencializando seus recursos internos para enfrentar as diversas etapas do tratamento com mais coragem, aderindo melhor ao mesmo e tornando-os agentes de suas escolhas e não apenas meros expectadores dos procedimentos.

\section{REFERÊNCIAS}

American Psychological Association. Division 38. (2016). Healthy, wealthy and weiss: A history of division 38 (Health Psychology). Retrived from https://societyforhealthpsychology. org/wp-content/uploads/2016/07/DivHistory.pdf.

American Society for Reproductive Medicine. (2013). Definitions of infertility and recurrent pregnancy loss: a committee opinion. Fertility and Sterility, 99(1), 63. https://doi.org/10.1016/j. fertnstert.2012.09.023

Balmaceda, R., Fernández, O., Fernández, E., Fabres, V., Huidobro, A., Sepúlveda, J., \& Zegers, F. (2001). Tener um hijo: Conociendo la infertilidad y los caminos para resolverla. Santiago: Publicações Técnicas Mediterrâneo.
Chaves, M. L. F. (n.d.). Testes de avaliação cognitiva: Mini-exame do estado mental. Retrieved from http://www.cadastro.abneuro. org/site/arquivos_cont/8.pdf

Cunha, J. A. (2000). A história do examinando. In J. A. Cunha (Org.), Psicodiagnostico - V(5 ed., pp. 57-66). Porto Alegre: Artmed.

Cunha, J. A. (2011). Manual da versão em português das Escalas Beck. São Paulo: Casa do Psicólogo.

Dalgalarrondo, P. (2008). Psicopatologia e semiologia dos transtornos mentais. Porto Alegre: Artmed.

Dornelles, L. M. N. (2009). Tornar-se Pai e Mãe no Contexto da Reprodução Assistida (Tese de Doutorado). Universidade Federal do Rio Grande do Sul, Porto Alegre, RS, Brasil. 
Folstein, M. F, Folstein, S. E, \& McHugh, P. R. (1975). “Mini-mental state". A practical method for grading the cognitive state of patients for the clinician. Journal of Psychiatric Research, 12(3), 189-198.

Klonoff-Cohen, H., Chu, E., Natarajan, L., \& Sieber, W. (2001). A prospective study of stress among women undergoing in vitro fertilization or gamete intrafallopian transfer. Fertility and Sterility, 76(4), 675-687.

Makuch, M.Y. (2006). Gênero e reprodução assistida: Novas fases e velhas questões. In R. M. M. Melamed \& J. Quayle (Orgs.), Psicologia em reprodução assistida: Experiências brasileiras (pp. 21-33). São Paulo: Casa do Psicólogo.

Melamed, R. M. M. (2013). A psicologia e o psicólogo na reprodução humana assistida. In I Consenso de Psicologia em Reprodução Assistida (pp. 23-29). São Paulo/Rio de Janeiro: Livre Expressão Editora. Makuch, M.Y.

Prosdocimo, A. C. M. G. (2011). Protocolos em psicologia hospitalar. In Anais do VIII Congresso da SBPH. Curitiba: Sociedade Brasileira de Psicologia Hospitalar.

Santos, J. R. S., \& Lanius, M. (2013). Intervenções psicológicas na infertilidade - Aconselhamento, terapias, outras aproximações terapêuticas. In I Consenso de Psicologia em Reprodução Assistida (pp. 39-45). São Paulo/Rio de Janeiro: Livre Expressão Editora.
Seger, L. (2009) Avaliação emocional do paciente de reprodução humana assistida (RHA). In R. Melamed, L. Seger, \& M. Borges Jr. (Orgs.), Psicologia e reprodução humana assistida (pp. 81-89). São Paulo: Livraria Santos Editora.

Seger, L., \& Melamed, R. M. M. (2011). A saúde e a doença na reprodução humana assistida. In E. Borges Jr., L. M. S. Farah \& S. S. Cortezzi (Orgs.), Reprodução humana assistida (pp. 289-294). São Paulo: Atheneu.

Tavares, M. (2000). A entrevista clínica. In J. A. Cunha (Org.), Psicodiagnostico - V ( $5^{\mathrm{a}}$ ed., pp. 45-56), Porto Alegre: Artmed.

Travain, A. A., Lourenço, G. V., \& Tardin, R. M. (2013). Avaliação psicológica do paciente infértil: Áreas a avaliar e instrumentos. In I Consenso de Psicologia em Reprodução Assistida (pp. 31-38). São Paulo/Rio de Janeiro: Livre Expressão Editora.

Submetido em: 26/09/2014

Revisado em: 19/07/2016

Aceito em: 09/08/2017 
Apêndice A. Fluxo de Atendimento do CEPRA

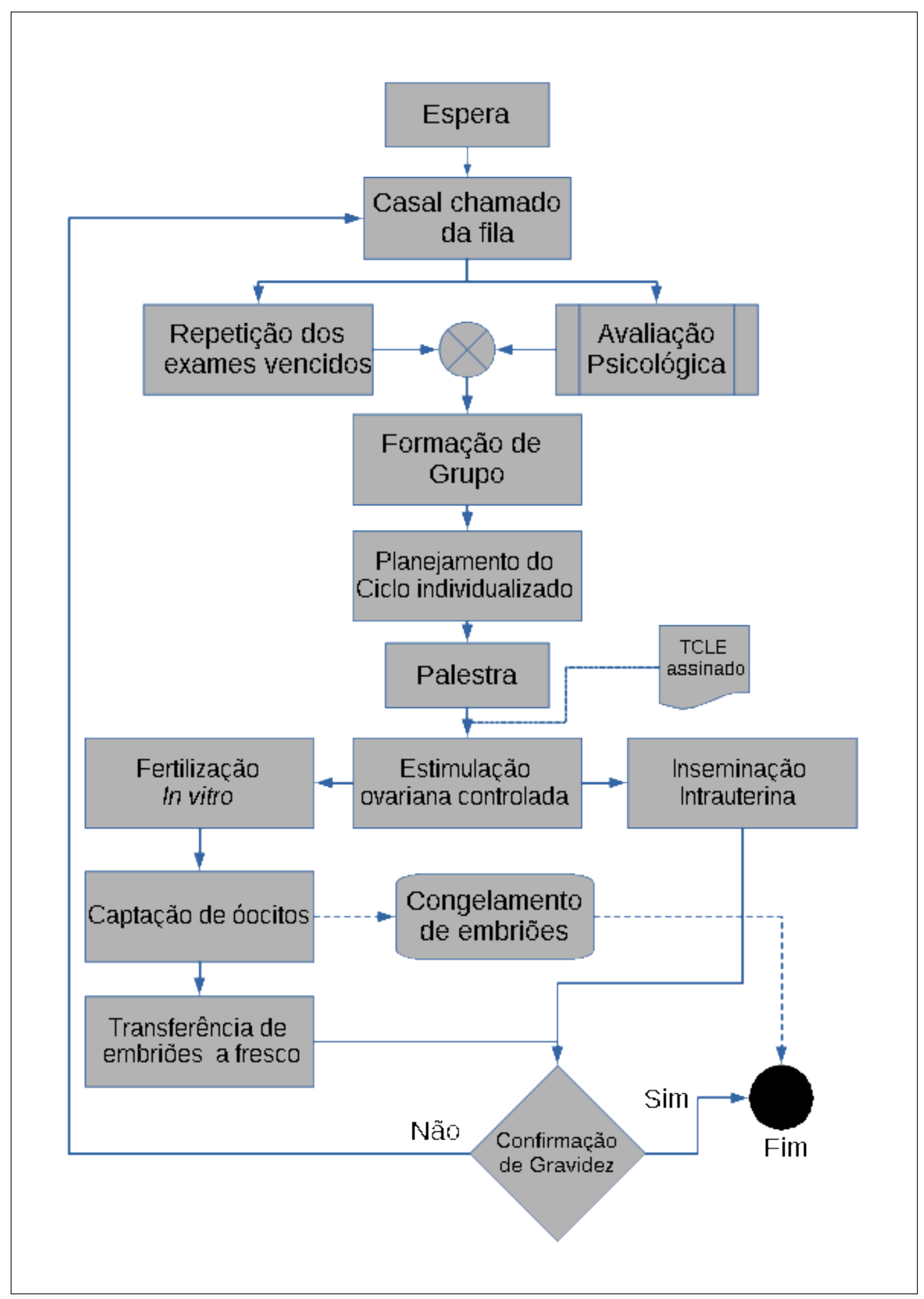


Apêndice B. Roteiro de entrevista semiestruturada de atendimento ao casal

ATENDIMENTO PSICOLÓGICO AO CASAL EM REPRODUÇÃO HUMANA ASSISTIDA

\begin{tabular}{|c|c|c|c|}
\hline & Sexo 1-M 2-F & & Sexo 1-M 2-F \\
\hline Nome & & Nome & \\
\hline \multicolumn{4}{|l|}{ Natural } \\
\hline Onde residem & D. N. & Natural & D. N. \\
\hline Profissão & & Profissão & \\
\hline Escolaridade & & Escolaridade & \\
\hline Religião & & Religião & \\
\hline
\end{tabular}

Juntos há

União do casal

Já têm filhos?

Tentam engravidar há

Tratamento a ser realizado ( ) IIU( )FIV( )TEC

Há quanto tempo aguardam na fila para o tratamento?

Possuem uma compreensão básica do tratamento e estão cientes das taxas de sucesso?

Causa(s) da infertilidade ( )fator feminino ( )fator masculino ( )sem causa aparente

Histórico obstétrico/ginecológico e tratamentos anteriores

Uso de cigarro/ bebida alcoólica/ drogas/ medicações em uso

Possuem rede de apoio social/ familiar? ( )SIM ( )NÃO

Como está a relação do casal?

Projetos de vida do casal

Possuem antecedentes psiquiátricos?

Grau de ansiedade

Humor deprimido ( )SIM ( )NÃO

Fatores geradores de estresse

Recursos de controle de estresse

Exame do estado mental

\section{PARECER}

Comportamentos/ sentimentos observados e possíveis encaminhamentos:

( ) APTOS ( ) NÃO APTOS DATA: 
Apêndice C. Fluxo de Atendimento da Psicologia do CEPRA

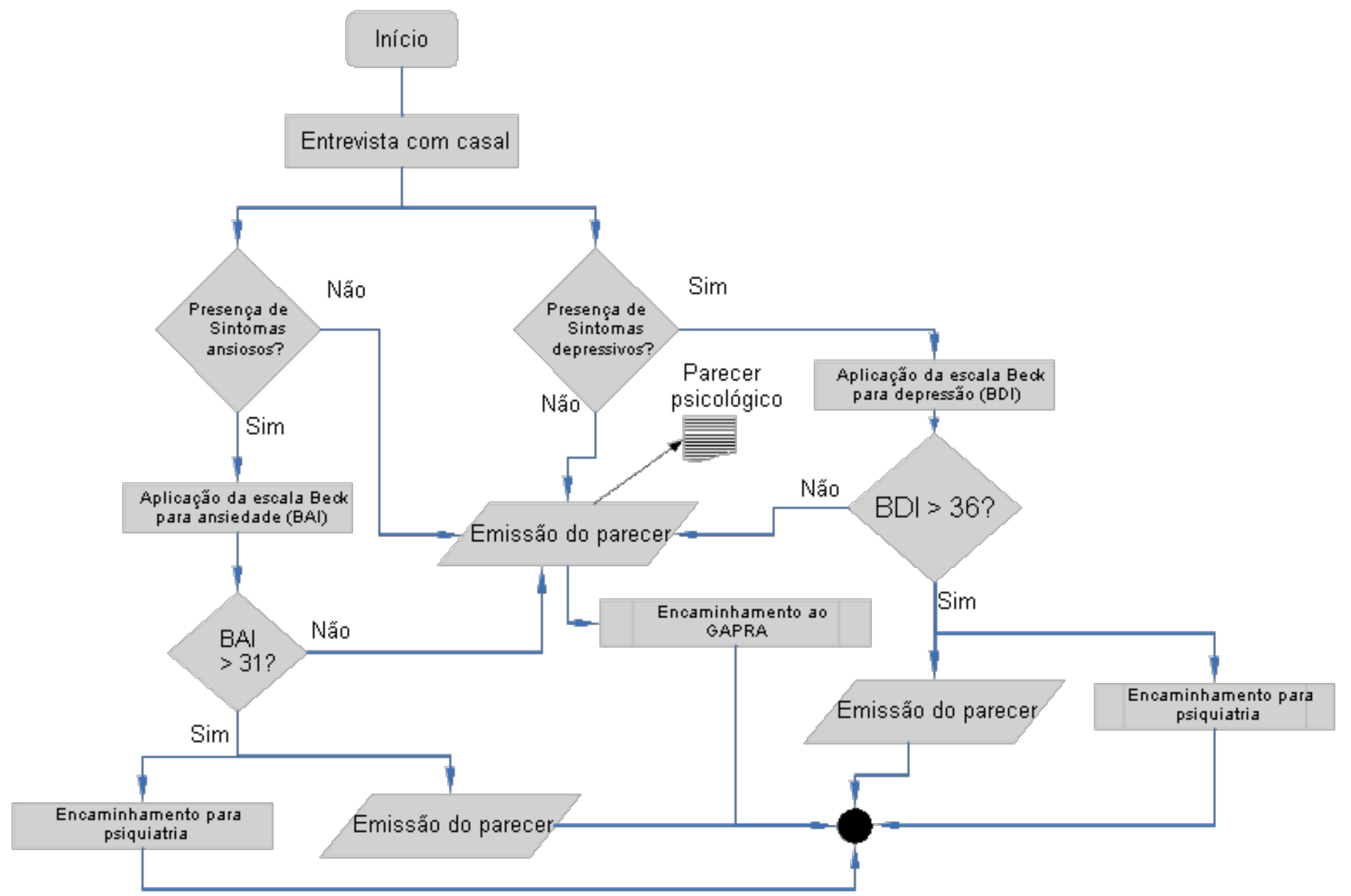

